\title{
Di una particolare teoria delle deformazioni elastiche finite.
}

\section{Nota di UMBERTO CRUDELI.}

I. - In un lavoro comparso nelle Göttinger Nachrichten ') del 1893 ed in uno successivo (1894) degli Annalen der Physik "), il Voigt si propose di estendere la teoria classica dell'elasticità, nel caso di solidi isotropi, assumendo, come potenziale elastico (specifico), tale una funzione razionale di terzo grado degl'invarianti fondamentali di deformazione che si traducesse in una funzione razionale di terzo grado delle caratteristiche di deformazione. Il secondo dei suddetti lavori avrebbe avuto per iscopo di mostrare la generica espressione del potenziale elastico, facendovi figurare tutti gl' invarianti fondamentali di deformazione (i quali, come è noto, sono in numero di tre) e di sviluppare, corrispondentemente, la proposta teoria, ciò che, a motivo di un teorema inesatto dello Schönflies ${ }^{3}$ ), egli non fece che parzialmente, $\theta$ con procedimento non corretto, nel primo di quei lavori medesimi. Lo scopo principale dei quali era certamente di applicare le nuove considerazioni alla deformazione dei cilindri per semplice trazione, affinchè potessero confrontarsi, in qualche caso concreto, le corrispondenti formule con quelle empiriche di J. O. Thompson "), le quali trovansi citate anche in qualche recente trattato di fisica $\left.{ }^{5}\right)$. Un tale confronto, però, non venne effettivamente eseguito. Comunque, il procedimento del Voigt richiede una completa correzione, non

1) (von der Gesell. der Wiss., pag. 534).

2) Pag. 536. - Vedısi anche: Sitzungsb. der Preus. Akad. der Wiss. zu Berlin., 1901, pag. 1266.

2) Vedasi: Göttinger Nachr., 1894, pag. 33,

4) Ann. der Phys, 1891, pag. 555.

s) Vedasi, p. es., il Trattato di fisica sperimentale di Battelli e Cardani, vol. I, pag. 283 . 
avendo egli tenuto presente che, qualora, in vista di una migliore teoria, si voglia assumere, come espressione del potenziale elastico, una funzione di grado superiore al secondo, rispetto alle caratteristiche di deformazione, non più è logico ritenere, come espressioni delle caratteristiche medesime, quelle che figurano nella teoria classica e che, inoltre, le componenti di tensione non più sono date dalle derivate del potenzialo rispetto alle corrispondenti caratteristiche della deformazione ${ }^{6}$ ).

Una prima idea che razionalmente si presenta allo studioso, intento ad ottenere un ulteriore approssimazione nello studio delle deformazioni elastiche, è certamente quella di ritenere, in uno sviluppo in serie del potenziale (considerato come funzione degli allungamenti principali), oltre i termini del secondo grado negli allungamenti medesimi, anche quelli cho immediatamente seguono, cioè anche i termini del terzo grado. Alcuni autori poi hanno proposto, formule da sostituire a quella classica di Hooke ${ }^{7}$ ) limitandosi alle deformazioni per semplice trazione ed a quelle per semplice compressione, senza, però, mostrare se e come le loro formule potevano entrare in uno schema generale di qualche nuova teoria dell'elasticità.

Io, qui, conservando per il potenziale l'espressione proposta dal Voigt, debitamente interpretata, e sfruttando le formule generali dell'Almansi ${ }^{8}$ ) relative alle tensioni elastiche, mi limiterò a correggere il procedimento del Voigt per ritrovare, nel caso di un cilindro rotondo soggetto a semplice trazione, l'allungamento del cilindro stesso in funzione della tensione che insiste sopra una sezzione normale all'asse.

II. - In corrispondenza di un generico punto del solido deformato, siano $a_{1}, a_{2}, a_{3}$ gli allungamenti principali $\theta$ si ponga

') Almansi, Rend. Accad. dei Lincei, 1911, I. e II. sem., e II. sem. 1917.

7) Vedasi, p. es.: Mehmke, Zum Gesetz der elastischen Dehnungen, Zeits. fil Math. und Phys., 1897, pag. 327.

F. Kohlrausch und T. Grüneisen, Sitzungsber. der Preuss. Akad. zu Berlin, 1901, pag. 1086.

A. Montel, Elasticilà e resistenza dei corpi pietrosi, Torino 1914, (Introduzione).

') Rend. Accad. dei Linvei, 1911. 


$$
\left\{\begin{array}{l}
\delta=a_{1}+a_{2}+a_{3} \\
\gamma=a_{1}^{2}+a_{3}^{2}+a_{3}^{2} \\
\zeta=a_{1} a_{2} a_{3} \\
2 \varphi=c_{1} \delta^{2}+c_{2} \gamma+\frac{2}{3} c_{1}^{\prime} \delta^{3}+c_{2}^{\prime} \delta \gamma+2 c_{3}^{\prime} \zeta,
\end{array}\right.
$$

denotando $\varphi$ il potenziale di elasticità e $c_{1}, c_{2}, c_{1}^{\prime}, c_{2}^{\prime}, c_{3}^{\prime}$ delle costanti ${ }^{\circ}$ ).

Le tensioni unitarie sopra elementi normali alle direzioni cai corrispondono gli allungamenti principali sono date dalle formule di Almansi

$$
\left\{\begin{array}{l}
\tau_{1}=\frac{1}{\left(1+a_{3}\right)\left(1+a_{3}\right)} \frac{\partial \varphi}{\partial a_{1}} \\
\tau_{2}=\frac{1}{\left(1+a_{3}\right)\left(1+a_{1}\right)} \frac{\partial \varphi}{\partial a_{2}} \\
\tau_{3}=\frac{1}{\left(1+a_{1}\right)\left(1+a_{9}\right)} \frac{\partial \varphi}{\partial a_{3}}
\end{array}\right.
$$

Talchè, nel nostro caso,

$$
\begin{array}{r}
\tau_{1}=\frac{1}{\left(1+a_{2}\right)\left(1+a_{3}\right)}\left\{c_{1} \delta+c_{2} a_{1}+c_{1}^{\prime} \delta^{2}+\right. \\
\left.\quad+c^{\prime}\left(a_{1} \delta+\frac{1}{2} \gamma\right)+c_{3}^{\prime} a_{2} a_{3}\right\},
\end{array}
$$

e le due analoghe.

') Giova notare che trattandosi qui di deformazioni faite, i tre invarianti di deformazione $\delta, \gamma, \zeta$ non coincidono coi fondumentali. 
Potremo anche scrivere

$$
\left\{\begin{array}{l}
\left(1+a_{3}\right)\left(1+a_{3}\right) \tau_{1}=\left(c_{1}+c_{2}\right) a_{1}+c_{1}\left(a_{2}+a_{3}\right)+T_{1} \\
\left(1+a_{3}\right)\left(1+a_{1}\right) \tau_{2}=\left(c_{1}+c_{2}\right) a_{2}+c_{1}\left(a_{3}+a_{1}\right)+T_{2} \\
\left(1+a_{1}\right)\left(1+a_{2}\right) \tau_{3}=\left(c_{1}+c_{2}\right) a_{3}+c_{1}\left(a_{1}+a_{3}\right)+T_{3}
\end{array}\right.
$$

dove

$$
\mathrm{T}_{1}=c_{1}^{\prime} \delta^{2}+c_{2}\left(a_{1} \delta+\frac{1}{2} \gamma\right)+c_{3}^{\prime} a_{2} a_{3}, \quad \mathrm{~T}_{2}=\text { etc. }
$$

Nel caso del cilindro rotondo (isotropo ed omogeneo) soggetto a semplice trazione, supporremo $a_{1}, a_{3}, a_{3}$ costanti in tutto il solido ed, inoltre, assumendo come direzione 1 la direzione dell'asse, verrà supposto $a_{9}=a_{3}, \quad \tau_{s}=\tau_{3}=0$. Quindi, denotando $\tau_{1}$ con $\tau, a_{1}$ con $a$ ed $a_{3}$ od $a_{3}$ con $a^{\prime}$, otterremo, dalle formule precedenti

$$
\begin{aligned}
\left(1+a^{\prime}\right)^{2} \tau & =\left(c_{1}+c_{2}\right) a+2 c_{1} a^{\prime}+\mathrm{T}_{1} \\
0 & =\left(c_{1}+c_{2}\right) a^{\prime}+c_{1}\left(a+a^{\prime}\right)+\mathrm{T}_{2}
\end{aligned}
$$

dove

(1)

$$
\begin{gathered}
\mathrm{T}_{3}=\left(c_{1}^{\prime}+\frac{3}{2} c_{2}^{\prime}\right) a^{2}+2\left(2 c_{1}^{\prime}+c_{2}^{\prime}\right) a a^{\prime}+ \\
+\left(4 c_{1}^{\prime}+c_{2}^{\prime}+c_{3}^{\prime}\right) a^{\prime} \\
\mathrm{T}_{3}=\left(c_{1}^{\prime}+\frac{1}{2} c_{2}^{\prime}\right) a^{2}+\left(4 c_{1}^{\prime}+c_{2}^{\prime}+c_{3}^{\prime}\right) a a^{\prime}+ \\
+\left(4 c_{1}^{\prime}+3 c_{2}^{\prime}\right) a^{\prime} .
\end{gathered}
$$

Ora si osservi che, denotando con $\sigma_{0}$ la primitiva sezione retta del eilindro e con $\sigma$ la sezione retta finale del cilindro medesimo si ha

$$
\left(1+a^{\prime}\right)^{2}=\frac{\sigma}{\sigma_{0}}
$$


talchè, ponendo $\tau \sigma=\tau_{0} \sigma_{0}$, potremo scrivere

$$
\left\{\begin{array}{l}
\tau_{0}=\left(c_{1}+c_{2}\right) a+2 c_{1} a^{\prime}+\mathrm{T}_{1} \\
0=\left(c_{1}+c_{2}\right) a^{\prime}+c_{1}\left(a+a^{\prime}\right)+\mathrm{T}_{2} ;
\end{array}\right.
$$

dalla seconda delle quali otteniamo

$$
a^{\prime}=-\frac{c_{1}}{2 c_{1}+c_{2}} a-\frac{1}{2 c_{1}+c_{2}} \mathrm{~T}_{1} .
$$

$\mathbf{E}$, ponendo

$$
\frac{c_{1}}{2 c_{1}+c_{2}}=\lambda \quad, \quad c_{2}=\frac{E}{1+\lambda},
$$

avremo

$$
a^{\prime}=-\lambda a-(1+\lambda)(1-2 \lambda) \frac{\mathrm{T}_{2}}{\mathrm{E}}
$$

La prima delle (2) potrà scriversi

$$
\tau_{0}=\mathrm{E} a+\mathrm{T}_{1}-2 \lambda \mathrm{T}_{2}
$$

da cui

$$
a=\frac{\tau_{0}}{\mathrm{E}}-\frac{1}{\mathrm{E}}\left(\mathrm{T}_{1}-2 \lambda \mathrm{T}_{2}\right)
$$

In prima approssimazione si ha la formula della tooria classica $a=\frac{\tau_{0}}{\mathrm{E}}$. In seconda approssimazione, tenendo $\operatorname{com} \theta$ espressioni di $\mathrm{T}_{1}$ e di $\mathrm{T}_{2}$ quelle che si ottengono col sostituire, dapprima, nelle (1), $-\lambda a$ ad $a^{\prime}$ e poi $\frac{\tau_{0}}{\mathrm{E}}$ ad $a$, avremo

$$
\begin{gathered}
a=\frac{\tau_{0}}{\mathrm{E}}-\frac{1}{2 \mathrm{E}^{3}}\left\{2(1-2 \lambda)^{3} c_{1}^{\prime}+3(1-2 \lambda)\left(2 \lambda^{2}+1\right) c_{2}^{\prime}+\right. \\
\left.+6 \lambda^{2} c_{3}^{\prime}\right\} \tau_{0}^{2},
\end{gathered}
$$


formula che coincide con quella del Voigt ${ }^{10}$ ). Infine, denotando con $h_{0}$ la primitiva lunghezza del cilindro e con $\nu$ il complessivo allangamento del cilindro medesimo, e. ponendo

$$
\left\{\begin{array}{c}
\tau_{0} \sigma_{0}=\mathrm{P}, \quad \frac{h_{0}}{\sigma_{0} \mathrm{E}}=\alpha \\
-\frac{1}{2 \sigma_{0}^{3} \mathrm{E}^{3}}\left\{2(1-2 \lambda)^{3} c_{1}^{\prime}+3(1-2 \lambda)\left(2 \lambda^{2}+1\right) c_{1}^{\prime}+\right. \\
\left.+6 \lambda^{2} c_{3}^{\prime}\right\}=\beta
\end{array}\right.
$$

avremo

$$
\nu=\alpha P+\beta P^{2}
$$

10) Annal. der Physik, 1894. Nella formula in discorso del Voigt, nella quale $\lambda$ ed $E$, dal Voigt non lntrodotti, sono espressi mediante $c_{1}$ e $c_{3}$, va rilevato un errore di stampa: al poșto di $c^{\prime} c_{2}$ va posto $c^{\prime} c_{3}{ }_{2}$. 